PROCEEDINGS OF THE

AMERICAN MATHEMATICAL SOCIETY

Volume 135, Number 7, July 2007, Pages 2257-2262

S 0002-9939(07)08736-9

Article electronically published on March 2, 2007

\title{
BURGERS EQUATION WITH RANDOM BOUNDARY CONDITIONS
}

\author{
YURI BAKHTIN \\ (Communicated by Edward C. Waymire)
}

\begin{abstract}
We prove an existence and uniqueness theorem for stationary solutions of the inviscid Burgers equation on a segment with random boundary conditions. We also prove exponential convergence to the stationary distribution.
\end{abstract}

\section{IntRoduCtion AND THE MAIN RESUlT}

The inviscid Burgers equation

$$
u_{t}+u u_{x}=0
$$

is one of the simplest nonlinear evolutionary PDEs.

In the absence of boundaries, solutions of (1) were studied extensively in mathematical and physical literature beginning with [2] and 4].

Many interesting questions arise if one perturbs the Burgers equation with random force. Existence and uniqueness of a stationary solution of the inviscid stochastically forced Burgers equation on a circle was shown in [3], and on multidimensional torus in [5]. Several other probabilistic problems for the Burgers equation are discussed in [7. However, random boundary conditions have not yet been studied. The goal of this note is to prove an existence and uniqueness theorem for stationary solutions of the Burgers equation on the segment $[0,1]$ with zero viscosity and random boundary conditions. Moreover, we prove exponential convergence to the stationary distribution of this system and the so-called One Force - One Solution Principle.

It is well-known that solutions of the initial value problem for equation (11) develop shocks in finite time and, generically, classical global solutions do not exist. However there are infinitely many solutions in the sense of distributions, and there is a unique entropy solution among them if the initial data grow not too fast. An entropy solution is a solution $u$ in the sense of distributions such that at each time $t$ and at any point $x$ the left and right limits $u(x \pm, t)$ exist and the entropy condition is satisfied:

$$
u(x-, t) \geq u(x+, t) .
$$

Received by the editors December 22, 2005 and, in revised form, March 30, 2006.

2000 Mathematics Subject Classification. Primary 35R60, 35Q53; Secondary 76M30.

Key words and phrases. Burgers equation, stationary solution, random boundary conditions.

(C)2007 American Mathematical Society Reverts to public domain 28 years from publication 2257 
We shall consider (1) on the segment $[0,1]$ with initial data

$$
u\left(x, t_{0}\right)=u_{0}(x), \quad x \in[0,1],
$$

and boundary conditions:

$$
\begin{array}{ll}
u(t, 0)=u_{L}(t), & t>t_{0} \\
u(t, 1)=u_{R}(t), & t>t_{0} .
\end{array}
$$

It is shown in 1 that there may be no piecewise continuous solution to the problem (10) - (5). This can be fixed if one replaces (4) and (5) by relaxed boundary conditions tightly related to the entropy condition (see [1] and 6]). Namely, we shall require that for almost all (with respect to the Lebesgue measure) $t>t_{0}$

$$
\begin{gathered}
u(0+, t)=u_{L}(t) \\
\text { or } \\
u(0+, t) \leq 0 \text { and }|u(0+, t)| \geq\left|u_{L}^{+}(t)\right|,
\end{gathered}
$$

and

$$
\begin{gathered}
u(1-, t)=u_{R}(t) \\
\text { or } \\
u(1-, t) \leq 0 \text { and }|u(1-, t)| \geq\left|u_{R}^{-}(t)\right|,
\end{gathered}
$$

where

$$
a^{+}=a \vee 0, \quad a^{-}=a \wedge 0, \quad a \in \mathbb{R} .
$$

We denote $\Pi_{t}=(0,1) \times(t, \infty)$ for $t \in \mathbb{R}$ and $\Pi=\Pi_{-\infty}=(0,1) \times \mathbb{R}$.

Theorem 1.1 (1]). Suppose that $u_{0}$ is measurable and bounded, and $u_{L}, u_{R}$ are measurable and locally bounded. Then there is a unique entropy solution of (1) in $\Pi_{t_{0}}$ satisfying (3), (6) and (7).

We shall denote by $u\left(x, t, u_{0}, t_{0}\right)$ the solution provided by this theorem at a point $(x, t) \in \Pi_{t_{0}}$.

We shall now assume that the boundary conditions $u_{L}$ and $u_{R}$ on the whole real time line are given by two standard independent Ornstein-Uhlenbeck (OU) processes, i.e. stationary Gaussian processes with zero mean and covariance function

$$
\operatorname{cov}\left(u_{L}(t), u_{L}(s)\right)=\operatorname{cov}\left(u_{R}(t), u_{R}(s)\right)=e^{-|t-s|},
$$

defined on a probability space $(\Omega, \mathcal{F}, \mathbb{P})$.

We can now state our main result.

Theorem 1.2. (1) With probability 1 there is a unique function $u: \Pi \rightarrow \mathbb{R}$ such that for any $t_{0}$ it is an entropy solution of (11) in $\Pi_{t_{0}}$ with initial data given by $u\left(\cdot, t_{0}\right)$ and boundary conditions (6), (7).

(2) One Force - One Solution Principle: With probability 1 the solution $u(\cdot, t)$ is uniquely defined by the history of the boundary conditions from $-\infty$ up to $t$. Namely, there is a functional $\Psi$ defined for almost every realization of a pair of standard independent $O U$ processes on $(-\infty, 0]$ such that

$$
u(\cdot, t)=\Psi\left(\pi_{t} u_{L}, \pi_{t} u_{R}\right),
$$

where $\pi_{t} v(s)=v(t+s)$ for $s \leq 0$.

(3) This solution $u$ is a stationary in time stochastic process. 
(4) Exponential convergence to the stationary distribution: there are constants $C, \lambda>0$ such that for any $t_{0}$, any initial data $u_{0}$ and any $t>t_{0}$,

$$
\mathbb{P}\left\{u(\cdot, t) \neq u\left(\cdot, t, u_{0}, t_{0}\right)\right\} \leq C e^{-\lambda\left(t-t_{0}\right)} .
$$

Remark. Our proof applies to a wide class of random boundary conditions. We take an OU process as the boundary condition for simplicity of presentation.

\section{Proof of the main Result}

Our proof of the main result is based on a variational principle for entropy solutions of mixed problems established in 6 . We now assume that the initial data are given at time $t_{0}$ and the conditions of Theorem 1.1. We shall explain how to construct the entropy solution of the problem (11), (3), (6), (7) at time $t>t_{0}$.

A continuous and piecewise linear map $\gamma:\left[t_{0}, t\right] \rightarrow[0,1]$ will be called a path on $\left[t_{0}, t\right]$. For any path there is a finite sequence of points

$$
\left(y_{0}, s_{0}\right),\left(y_{1}, s_{1}\right), \ldots,\left(y_{n}, s_{n}\right)
$$

in $[0,1] \times\left[t_{0}, t\right]$ such that $t_{0}=s_{0}<s_{1}<\ldots<s_{n}=t$, and for each $k=0, \ldots, n-1$ the path is given by

$$
\gamma(s)=y_{k}+\frac{s-s_{k}}{s_{k+1}-s_{k}}\left(y_{k+1}-y_{k}\right), \quad s \in\left[s_{k}, s_{k+1}\right] .
$$

These points $\left(y_{k}, s_{k}\right)$ are called vertices of the path. Though they define the path uniquely, they are not uniquely defined by the path (one can insert a new vertex between any two subsequent vertices without altering the path). However, it is sometimes convenient to identify a path with its vertices. It is also convenient to consider a path as a curve in $\bar{\Pi}=[0,1] \times \mathbb{R}$. In fact, it is a polyline composed of segments connecting vertices of the path. For two points $A, B \in \bar{\Pi}$ we shall denote the line segment connecting them by $[A B]$.

For every path $\gamma$ we define its action functional as

$$
S(\gamma)=\frac{1}{2} \int_{\gamma(s) \in(0,1)} \dot{\gamma}^{2}(s) d s-\frac{1}{2} \int_{\gamma(s)=0} u_{L}^{+2}(s) d s-\frac{1}{2} \int_{\gamma(s)=1} u_{R}^{-2}(s) d s .
$$

Here $a^{+}=a \wedge 0, a^{-}=a \vee 0$ for a number $a \in \mathbb{R}$.

For $x \in(0,1)$ the set of all paths $\gamma$ on $\left[t_{0}, t\right]$ with $\gamma(t)=x$ is denoted by $\Gamma\left(t_{0} ; x, t\right)$. Let us define

$$
S\left(t_{0} ; x, t\right)=\inf _{\gamma \in \Gamma\left(t_{0} ; x, t\right)}\left[S(\gamma)-U_{0}\left(\gamma\left(t_{0}\right)\right)\right]
$$

where

$$
U_{0}(x)=\int_{0}^{x} u_{0}(y) d y .
$$

Theorem 2.1 ([6]). The following holds true for any $t_{0}$ :

(1) For each $x, t \in \Pi_{t_{0}}$ there is a minimizing path $\gamma^{*}$ in the definition of the function $S\left(t_{0} ; x, t\right)$, i.e. the set $\Gamma^{*}\left(t_{0} ; x, t\right)$ of minimizing paths is not empty.

(2) $S\left(t_{0} ; x, t\right)$ is a.e. differentiable in $x$, and

$$
u(x, t)=\frac{\partial S\left(t_{0} ; x, t\right)}{\partial x}
$$

is an entropy solution of (11), (3), (6), (7). 
(3) For each fixed $(x, t) \in \Pi_{t_{0}}$

$$
\begin{aligned}
& u(x-, t)=\sup _{\gamma \in \Gamma^{*}\left(t_{0} ; x, t\right)} \dot{\gamma}(t), \\
& u(x+, t)=\inf _{\gamma \in \Gamma^{*}\left(t_{0} ; x, t\right)} \dot{\gamma}(t),
\end{aligned}
$$

and these extremal values are attained at some paths $\gamma_{-}$and $\gamma_{+}$respectively.

Remark. It is clear from the definition of the action functional that action-minimizing paths tend to spend a lot of time on the boundary, thus accumulating negative action. A more detailed description of the structure of these paths will be given in Lemma 2.1 below.

We shall need some terminology. Any minimizing path from part (11) of Theorem 2.1 will be called a $\left(t_{0} ; x, t\right)$-minimizer. Minimizing paths $\gamma_{-}$and $\gamma_{+}$in part (3) will be called left and right $\left(t_{0} ; x, t\right)$-minimizers respectively.

We shall say that two points $A_{1}=\left(x_{1}, t_{1}\right), A_{2}=\left(x_{2}, t_{2}\right) \in \bar{\Pi}$ are connected by a path $\gamma$ if they belong to this path and $t_{1}<t_{2}$. For any two points $\left(x_{1}, t_{1}\right)$ and $\left(x_{2}, t_{2}\right)$ with $t_{1}<t_{2}$ the set of all paths connecting $\left(x_{1}, t_{1}\right)$ and $\left(x_{2}, t_{2}\right)$ will be denoted by $\Gamma\left(x_{1}, t_{1} ; x_{2}, t_{2}\right)$. Any path providing minimum in the variational problem

$$
S(\gamma) \rightarrow \min , \quad \gamma \in \Gamma\left(x_{1}, t_{1} ; x_{2}, t_{2}\right)
$$

is called an $\left(x_{1}, t_{1} ; x_{2}, t_{2}\right)$-minimizer. The set of all $\left(x_{1}, t_{1} ; x_{2}, t_{2}\right)$-minimizers is called $\Gamma^{*}\left(x_{1}, t_{1} ; x_{2}, t_{2}\right)$. Paths solving

$$
\begin{aligned}
& \dot{\gamma}\left(t_{2}\right) \rightarrow \max , \quad \gamma \in \Gamma^{*}\left(x_{1}, t_{1} ; x_{2}, t_{2}\right), \\
& \dot{\gamma}\left(t_{2}\right) \rightarrow \min , \quad \gamma \in \Gamma^{*}\left(x_{1}, t_{1} ; x_{2}, t_{2}\right),
\end{aligned}
$$

are called left and right $\left(x_{1}, t_{1} ; x_{2}, t_{2}\right)$-minimizers respectively.

Let $A_{1}=\left(x_{1}, t_{1}\right), \ldots, A_{n}=\left(x_{n}, t_{n}\right)$ be a sequence of points such that points $A_{k}, A_{k+1}$ are connected by a path $\gamma_{k}$ for $k=1, \ldots, n-1$. We shall use the notation $A_{1} \gamma_{1} A_{2} \gamma_{2} \ldots \gamma_{n-1} A_{n}$ to denote a new path composed of restrictions of paths $\gamma_{k}$ on segments $\left[t_{k}, t_{k+1}\right]$.

Suppose two points $A$ and $B$ are connected by two paths $\gamma_{1}$ and $\gamma_{2}$. We say that paths $A \gamma_{1} B$ and $A \gamma_{2} B$ are interchangeable if $S\left(A \gamma_{1} B\right)=S\left(A \gamma_{2} B\right)$.

First, we need the following basic result on the first term in the definition of $S(\gamma)$.

Lemma 2.1. $\quad$ (1) Suppose a path $\gamma$ is linear between $t_{1}$ and $t_{2}$. Then

$$
\frac{1}{2} \int_{t_{1}}^{t_{2}} \dot{\gamma}^{2}(s) d s=\frac{\left(\gamma\left(t_{2}\right)-\gamma\left(t_{1}\right)\right)^{2}}{2\left(t_{2}-t_{1}\right)} .
$$

(2) ("Cutting Corners") If $t_{1}<t_{2}<t_{3}$, then for any $x_{1}, x_{2}, x_{3}$

$$
\frac{\left(x_{3}-x_{1}\right)^{2}}{2\left(t_{3}-t_{1}\right)} \leq \frac{\left(x_{3}-x_{2}\right)^{2}}{2\left(t_{3}-t_{2}\right)}+\frac{\left(x_{2}-x_{1}\right)^{2}}{2\left(t_{2}-t_{1}\right)},
$$

equality being attained if and only if the point $\left(x_{2}, t_{2}\right)$ belongs to the segment $\left[\left(x_{1}, t_{1}\right),\left(x_{3}, t_{3}\right)\right]$.

(3) If $\gamma \in \Gamma^{*}\left(x_{1}, t_{1} ; x_{2}, t_{2}\right)$ for some $x_{1}, t_{1}, x_{2}, t_{2}$, then each segment of $\gamma$ either has $\left(x_{1}, t_{1}\right)$ or $\left(x_{2}, t_{2}\right)$ as a vertex, or connects two boundary points of $\bar{\Pi}$.

Proof. The first statement is straightforward. To prove the second one it is sufficient to fix $t_{1}, t_{2}, t_{3}, x_{1}, x_{3}$ and find the minimum of the r.h.s. of (10) as a function of $x_{2}$. The third part follows from parts (1) and (2). 
Lemma 2.2. With probability 1 there is a sequence of times $\left(\tau_{n}\right)_{n \in \mathbb{N}}$ approaching $-\infty$ such that for each $n$ there is a $\left(0, \tau_{n} ; 0, \tau_{n}+1\right)$-minimizer $h_{n}$ with $h_{n}(s)=1$ for some $s \in\left[\tau_{n}, \tau_{n}+1\right]$.

Proof. Consider a time $r$ and a $(0, r ; 0, r+1)$-minimizer $h$. Due to Lemma 2.1 there may be two options. Either $h(s)=0$ for all $s \in[r, r+1]$, or $h(s)=1$ for some $s \in[r, r+1]$. The former is impossible on the event

$$
B_{r}=\left\{-\frac{1}{2} \int_{r}^{r+1} u_{L}^{+2}(\tau) d \tau>2 \cdot \frac{1}{2 \cdot 1 / 3}-\frac{1}{2} \int_{r+1 / 3}^{r+2 / 3} u_{R}^{-2}(\tau) d \tau\right\},
$$

since the r.h.s. of the inequality defining $B_{r}$ is the action functional of the path with vertices $(0, r),(1, r+1 / 3),(1, r+2 / 3),(0, r+1)$. Since $\mathbb{P}\left(B_{r}\right)>0$ due to the properties of Gaussian processes, and the boundary conditions are given by an ergodic stationary process, we conclude that with probability 1 infinitely many events $B_{r}$ happen and the proof is complete.

Lemma 2.3. Suppose $\gamma$ is an $\left(x_{1}, t_{1} ; x_{2}, t_{2}\right)$-minimizer or $\left(t_{1} ; x_{2}, t_{2}\right)$-minimizer, and $\gamma\left(s_{1}\right)=y_{1}, \gamma\left(s_{2}\right)=y_{2}$ for some $s_{1}, s_{2} \in\left[t_{1}, t_{2}\right]$. Then the restriction of $\gamma$ onto $\left[s_{1}, s_{2}\right]$ is a $\left(y_{1}, s_{1} ; y_{2}, s_{2}\right)$-minimizer. Moreover, if $\gamma$ is a left or right minimizer, then so is the restriction of $\gamma$.

Proof. This obvious statement is a consequence of Bellman's optimality principle.

Lemma 2.4. Suppose two minimizers $\gamma_{1}$ and $\gamma_{2}$ have two common points $A=$ $\left(x_{1}, t_{1}\right)$ and $B=\left(x_{2}, t_{2}\right)$ with $t_{1}<t_{2}$. Then $A \gamma_{1} B$ and $A \gamma_{2} B$ are interchangeable.

Proof. Lemma 2.3 implies that both $A \gamma_{1} B$ and $A \gamma_{2} B$ are minimizers. Therefore, $S\left(A \gamma_{1} B\right)=S\left(A \gamma_{2} B\right)$.

Lemma 2.5. Suppose that for some $\tau$ there is a $(0, \tau ; 0, \tau+1)$-minimizer $\eta$ with $\eta(s)=1$ for some $s \in[\tau, \tau+1]$. For any point $E=(x, t) \in \Pi_{\tau+1}$ there are two numbers $u_{+}$and $u_{-}$with the following properties:

For any time $t_{0}<\tau$, any initial data $u_{0}$ at time $t_{0}$ and any right $\left(t_{0} ; x, t\right)$ minimizer $\gamma_{+}$,

$$
\dot{\gamma}_{+}(t)=u_{+}
$$

For any time $t_{0}<\tau$, any initial data $u_{0}$ at time $t_{0}$ and any left $\left(t_{0} ; x, t\right)$ minimizer $\gamma_{-}$,

$$
\dot{\gamma}_{-}(t)=u_{-} .
$$

This lemma says that at time $\tau+1$ our system "does not remember" its evolution before time $\tau$, and its further evolution is fully determined by the boundary conditions after $\tau$.

Proof. It is clear that $\eta$ contains segments $a=\left[\left(0, s_{1}\right),\left(1, s_{2}\right)\right]$ and $b=\left[\left(1, s_{3}\right),\left(0, s_{4}\right)\right]$ for some $s_{1}<s_{2}<s_{3}<s_{4}$. Consider any initial data $u_{0}$ and a right(left) $\left(t_{0} ; x, t\right)$ minimizer $\gamma$ connecting $A=\left(x_{0}, t_{0}\right)$ and $E$. Let $\gamma$ intersect $a$ and $b$ at points $B$ and $D$ respectively.

Lemma 2.4 implies that $B \eta D$ and $B \gamma D$ are interchangeable. Therefore, the path $A \gamma B \eta D \gamma E$ is also a minimizer containing $C=\left(1, s_{2}\right)$. Due to Lemma 2.3 $C \eta D \gamma E$ is a right(left) $\left(1, s_{2} ; x, t\right)$-minimizer. Therefore, $\dot{\gamma}(t)$ is uniquely defined and does not depend on initial data. 
Proof of the main result. According to Lemmas 2.2 and 2.5. with probability 1 there is a sequence of times $\tau_{n} \rightarrow-\infty$ such that for each $n$ the solution after time $\tau_{n}+1$ is uniquely defined by the boundary conditions. Therefore, the solution $u$ is uniquely defined for all $t \in \mathbb{R}$, and part (1) of the theorem is proven.

For any time $t$ this construction gives $u(\cdot, t)$ as a functional of the realization of the boundary conditions from $-\infty$ up to $t$. Let us call this functional $\Phi_{t}\left(\left.u_{L}\right|_{(-\infty, t]},\left.u_{R}\right|_{(-\infty, t]}\right)$, where $\left.u_{L}\right|_{(-\infty, t]}$ and $\left.u_{R}\right|_{(-\infty, t]}$ denote the realizations of $u_{L}$ and $u_{R}$ on $(-\infty, t]$. Clearly, the functional $\Phi_{t}$ is translationally invariant in time:

$$
\Phi_{t}\left(\left.u_{L}\right|_{(-\infty, t]},\left.u_{R}\right|_{(-\infty, t]}\right)=\Phi_{0}\left(\pi_{t} u_{L}, \pi_{t} u_{R}\right) .
$$

Therefore, we can denote $\Psi=\Phi_{0}$, and the proof of part (2) is complete.

Since $u$ is a functional of a stationary process it is a stationary process itself, and part (3) is proven.

Next, we notice that

$$
\left\{u(\cdot, t) \neq u\left(\cdot, t, u_{0}, t_{0}\right)\right\} \subset \bigcap_{t_{0}<r<t-1} B_{r}^{c},
$$

where $B_{r}$ is defined in (11). Now part (44) follows from $\mathbb{P}\left(B_{r}\right)>0$ since the boundary conditions are given by a geometrically recurrent Markov stationary process.

\section{REFERENCES}

1. C. Bardos, A. Y. le Roux, and J.-C. Nédélec, First order quasilinear equations with boundary conditions, Comm. Partial Differential Equations 4 (1979), no. 9, 1017-1034. MR0542510 (81b:35052)

2. J. M. Burgers, The nonlinear diffusion equation: asymptotic solutions and statistical problems, D. Reidel Pub. Co., 1974.

3. Weinan E, K. Khanin, A. Mazel, and Ya. Sinai, Invariant measures for Burgers equation with stochastic forcing, Ann. of Math. (2) 151 (2000), no. 3, 877-960. MR1779561 (2002e:37134)

4. E. Hopf, The partial differential equation $u_{t}+u u_{x}=\mu u_{x x}$, Comm. Pure Appl. Math. 3 (1950), 201-230. MR0047234 (13:846c)

5. R. Iturriaga and K. Khanin, Burgers turbulence and random Lagrangian systems, Comm. Math. Phys. 232 (2003), no. 3, 377-428. MR1952472(2004b:76079)

6. K. T. Joseph and G. D. Veerappa Gowda, Solution of convex conservation laws in a strip, Proc. Indian Acad. Sci. Math. Sci. 102 (1992), no. 1, 29-47. MR 1163972 (93f:35142)

7. W. A. Woyczyński, Burgers $K P Z$ turbulence, Lecture Notes in Mathematics, vol. 1700, Springer-Verlag, Berlin, 1998, Göttingen lectures. MR1732301(2000j:60077)

The Fields Institute for Research in Mathematical Sciences, 222 College Street, Second Floor, Toronto, Ontario, Canada M5T 3J1

Current address: School of Mathematics, Georgia Institute of Technology, Atlanta, Georgia 30332-0160

E-mail address: ybakhtin@fields.utoronto.ca, bakhtin@math.gatech.edu 